\title{
FDA-defined tobacco product
}

Addiction Ontology

\section{Source}

Addiction Ontology

Definition: A product that is classified by the US Food and Drug Administration as a tobacco product.

Comment: An FDA defined tobacco product is any product that meets the definition of a tobacco product set out in the United States Food, Drug and Cosmetic Act and as interpreted by the courts. It is any product made or derived from tobacco that is intended for human consumption, including any component, part, or accessory of a tobacco product (except for raw materials other than tobacco used in manufacturing a component, part, or accessory of a tobacco product). It does not include an article that is a drug. This class is not coherent and is best avoided in scientific discourse because, despite the label, it includes products that have no tobacco constituents in them (e.g. e-cigarettes whose e-liquid does not contain nicotine) and excludes products that do have the tobacco constituent nicotine (e.g. nicotine gum).

Curator note: This class is required because it is used by US the Food and Drug Administration's Center for Tobacco Products to classify products over which it has jurisdiction.

This definition was imported from the Addiction Ontology (see https://addictovocab.org/). Comments and suggestions for improvements are welcome using the Qeios review system.

Definitions imported from the Addiction Ontology (AddictO) are what are known as 'ontological definitions'. See this article in Qeios for an explanation https://www.qeios.com/read/YGIF9B.

Ontological definitions can sometimes be hard to read. In those cases we also include an informal definition.

Definitions also often require elaboration to make it clear how they should be used and what they include. In those cases we include a comment.

Also, definitions sometimes require an explanation as to how they came about to help users understand how they relate to alternative definitions. In those cases we include a curator note. 
